\title{
A Prize Problem in Coding Theory
}

\author{
Jon-Lark Kim \\ Department of Mathematics, University of Louisville, Louisville, KY 40292, USA \\ jl.kim@louisville.edu
}

Summary. In this short note, we describe one of the long-standing open problems in algebraic coding theory, i.e., whether there exists a binary self-dual $[72,36,16]$ code.

\section{Introduction}

Binary self-dual codes or self-dual codes over finite fields in general have been of great interest partly because many good linear block codes are either selforthogonal or self-dual. It turns out that they satisfy a nonconstructive lower bound, analogous to the Gilbert-Varshamov bound in linear codes. Furthermore, they have nice algebraic properties; in particular, the weight enumerator of a self-dual code over a finite field is invariant under a certain finite matrix group, which further restricts the minimum distance of a self-dual code over $G F(2), G F(3)$, or $G F(4)$. We refer to [13], [9] for a full discussion of self-dual codes.

A binary self-dual code $C$ under the usual inner product is called a Type $I I$ (or doubly-even) code if all codewords have weight $\equiv 0 \quad(\bmod 4)$, and a Type $I$ (or singly-even) code if there is a codeword whose weight $\equiv 2(\bmod 4)$. Given a binary Type I code $C$, one can obtain the doubly-even subcode $C_{0}$ of $C$ (consisting of all codewords whose weight $\equiv 0 \quad(\bmod 4))$. The shadow $S$ of $C$ is defined by $S:=C_{0}^{\perp} \backslash C[1]$. The weight enumerator $S(x, y)$ of the shadow of $C$ is determined by the weight enumerator $C(x, y)$ of $C$ as $S(x, y)=$ $\frac{1}{|C|} C(x+y, i(x-y))$, where $i=\sqrt{-1}$. This additional relation gives a further restriction on a possible weight enumerator of a binary self-dual code, often proving the nonexistence of a putative binary self-dual code [1].

Using $C(x, y)$ and $S(x, y)$ in a sophisticated way, Rains [12] derived a tight upper bound on the minimum distance of a binary self-dual code. More precisely, if $C$ is a binary self-dual code of length $n$ with minimum distance $d$ then $d \leq 4\lfloor n / 24\rfloor+4$ except when $n \equiv 22 \quad(\bmod 24)$, in which case $d \leq$ $4\lfloor n / 24\rfloor+6($ see $[12])$. Further if $C$ is a Type I code of length $n \equiv 0 \quad(\bmod 24)$, 
then $d \leq 4\lfloor n / 24\rfloor+2$. A Type I self-dual code whose minimum distance $d$ attains this bound is called extremal. A Type II code of length $n$ with minimum distance $d=4\lfloor n / 24\rfloor+4$ is called extremal.

It has been one of important problems in coding theory to find (binary) extremal self-dual codes (see [6] for recent results on extremal self-dual codes over $G F(2), G F(3), G F(4), Z_{4}, G F(2)+u G F(2)$, and $\left.G F(2)+v G F(2)\right)$, due to their connection with other mathematical areas including designs, lattices, and modular forms [11], [9].

In particular, one of the most famous open problems is the following.

Problem : Does there exist a Type II $[24 k, 12 k, 4 k+4]$ code $C(k)$ for $k \geq 3$ ?

We note the following results.

1. If $k=1$, then $C(1)$ is the Type II $[24,12,8]$ code (the binary extended Golay code). In fact, any binary linear code with parameters $[24,12,8]$ is equivalent to $C(1)$ (Pless, $1968[10]$ ).

2. If $k=2$, then $C(2)$ is the extended quadratic residue code $X Q_{47}$ of length 48. This is unique up to equivalence among self-dual codes with parameters [48, 24, 12] (Houghten, Lam, Thiel, and Parker, 2003 [5]). It is not known whether there is a linear binary $[48,24,12]$ code other than $X Q_{47}$.

3. The existence of a Type II $[72,36,16]$ code $C(3)$ is one of the long-standing open problems in coding theory. This was officially suggested by Sloane in 1973 [14]. If it exists, then the codewords of weight 16 form a $5-(72,16,78)$ design whose existence is unknown.

4. If $k \geq 154$, then $C(k)$ does not exist since $A_{4 k+8}$ (the number of codewords of weight $4 k+8)$ is negative $([15])$.

\section{Related facts about a putative Type II $[72,36,16]$ code}

The weight enumerator of a putative Type II $[72,36,16]$ code $C(3)$ is given as follows.

$$
W=1+249,849 y^{16}+18,106,704 y^{20}+462,962,955 y^{24}+4,397,342,400 y^{28}+
$$
$+16,602,715,899 y^{32}+25,756,721,120 y^{36}+\cdots$.

One possible attack to prove or disprove the existence of $C(3)$ is to investigate the order of the automorphism group of $C(3)$. The only possible prime orders of an automorphism of $C(3)$ are 2,3,5, and 7. It is remarked [6] that Yorgov recently proved that the automorphism group has order a divisor of 72 or order $504,252,56,14,7,360,180,60,30,10$, or 5 .

Another attack is to construct codes related to $C(3)$. The existence of $C(3)$ is equivalent to that of a Type I [70,35,14] code (Rains, 1998 [12]). The weight enumerator of a Type I $[70,35,14]$ code is corrected in $[6]$ as follows:

$W=1+11,730 y^{14}+150,535 y^{16}+1,345,960 y^{18}+\cdots$. 
Gulliver, Harada, and Kim [4] showed that the existence of $C(k)$ implies the existence of a Type I $[24 k, 12 k, 4 k+2]$ code for $k \geq 1$. Hence if there is $C(3)$, then there is a Type I $[72,36,14]$ code. Equivalently, if there is no Type I $[72,36,14]$ code, there is no $C(3)$. No self-dual codes with parameters $[72,36,14]$ are known to exist. There are exactly three possible weight enumerators for a Type I $[72,36,14]$ code as follows.

$$
\begin{aligned}
& W_{1}=1+7616 y^{14}+134,521 y^{16}+1,151,040 y^{18}+\cdots, \\
& W_{2}=1+8576 y^{14}+124,665 y^{16}+1,206,912 y^{18}+\cdots \\
& W_{3}=1+8640 y^{14}+124,281 y^{16}+1,207,360 y^{18}+\cdots
\end{aligned}
$$

\section{Future work}

There is a hope that $C(3)$ might exist. For example, although it is not known yet whether there exists a binary linear $[72,36,16]$ code, there is a $[72,36,15]$ code by puncturing a $[73,36,16]$ cyclic code and any $[72,36, d]$ code satisfies $d \leq 17$ from Brouwer's Table.

A recent attempt to construct $C(3)$ was made by Dougherty, Kim, and Solé [2] by considering double circulant codes based on strongly regular graphs and doubly regular tournaments. In particular, SRG (Strongly Regular Graphs) with parameters $(36,15,6,6)$ produce a lot of Type II $[72,36,12]$ codes. Similarly DRT (Doubly Regular Tournaments) of order 36 produce Type II $[72,36,8$ or 12$]$ codes. It is hoped that $d=16$ is possible if there is enough data for DRT of the above parameters.

Furthermore, recently we [7] have shown that skew Hadamard matrices of order $4 m$ where a prime $p$ divides $m$ produce self-dual codes over $G F(p)$. In particular, if $m=18$, then we have plenty of Type II $[72,36,12]$ codes with various weight enumerators from the 990 skew Hadamard matrices of order 72 in [8]. This motivates an active search for more skew Hadamard matrices of order 72 .

From the viewpoint of the Groebner basis, it is shown [3] how to construct the input basis of a zero-dimensional polynomial ideal, whose solutions correspond to binary systematic non-linear codes with fixed parameters (length, dimension, and distance). It is obvious how to specialize it to classify binary linear codes. By computing the Groebner basis $G$ of Guerrini-Sala's ideal $B$ for parameters $[72,36,16]$, we would immediately have a complete classification for such codes, if they exist. In particular, if $G$ turns out to be trivial $(G=\{1\})$, then there are no such codes. If it is not trivial, its solutions can be tested whether they are self-dual. However, it is well possible that the computation of $G$ is infeasible, since $I$ has $36^{2}=1296$ variables.

\section{Monetary Prizes}

As far as we know, the existence of $C(3)$ is the only coding problem with monetary prizes. The detail can be found from 
http://academic.scranton.edu/faculty/doughertys1/

- N.J.A. Sloane offers $\$ 10$ (1973) - still valid (confirmed in 2006)

- F.J. MacWilliams offered $\$ 10$ (1977) - invalid now.

The following monetary prizes were announced in the Yamagata conference, October, 2000, and at WCC2001 in Paris.

- S.T. Dougherty offers $\$ 100$ for the existence of $C(3)$.

- M. Harada offers $\$ 200$ for the nonexistence of $C(3)$.

The prize is awarded only once and the result must be published in a refereed reputable mathematics journal. All decisions about the prize are decided by those offering the prize.

Acknowledgement: The author would like to thank Dr. Massimiliano Sala for his remark on the Groebner basis in Section 3.

\section{References}

1. Conway JH, Sloane NJA (1990) A new upper bound on the minimal distance of self-dual codes. IEEE Trans. Inform. Theory 36:1319-1333.

2. Dougherty ST, Kim J-L, Solé P (2007) Double circulant codes from two class association schemes. Advances in Mathematics of Communications 1:45-64.

3. Guerrini E, Sala M (2007) An algebraic approach to the classification of some non-linear codes. Workshop on Coding and Cryptography 2007, INRIA, 177185.

4. Gulliver TA, Harada M, Kim J-L (2003) Construction of some extremal selfdual codes. Discrete Math 263:81-91.

5. Houghten SK, Lam CWH, Thiel LH, Parker JA (2003) The extended quadratic residue code is the only $(48,24,12)$ self-dual doubly-even code. IEEE Trans. Inform. Theory 49:53-59.

6. Huffman WC (2005) On the classification and enumeration of self-dual codes. Finite Fields and Their Applications 11:451-490.

7. Kim J-L and Solé P (2008) Skew Hadamard designs and their codes, to appear in Designs, Codes, and Cryptography.

8. Kotsireas I, http://www.medicis.polytechnique.fr/ kotsirea/

9. Nebe G, Rains EM, Sloane NJA (2006) Self-Dual Codes and Invariant Theory, Series: Algorithms and Computation in Mathematics, vol. 17, Berlin, Springer.

10. Pless V(1968) On the uniqueness of the Golay codes. J. Combin. Theory, 5: 215-228.

11. Pless VS, Huffman WC, Eds. Handbook of Coding Theory, Amsterdam. The Netherlands: Elsevier, 1998.

12. Rains EM (1998) Shadow bounds for self-dual codes. IEEE Trans. Inform. Theory, 44:134-139.

13. Rains EM, Sloane NJA (1998) Self-dual codes. in Handbook of Coding Theory, ed. V. S. Pless and W. C. Huffman. Amsterdam: Elsevier, pp. 177-294.

14. Sloane NJA $(1973)$ Is there a $(72,36) d=16$ self-dual code. IEEE Trans. Inform. Theory 19:251.

15. Zhang S (1999) On the nonexistence of extremal self-dual codes. Discrete Appl. Math. 91:277-286. 